\title{
Occult alveolar haemorrhage in bronchopulmonary Kaposi's sarcoma
}

\author{
L Hughes-Davies, G Kocjan, M F Spittle, R F Miller
}

\begin{abstract}
Evidence of occult alveolar haemorrhage was sought by Perls's staining of bronchoalveolar lavage fluid to detect haemosiderin laden macrophages in 63 human immunodeficiency virus positive (HIV-1) men who underwent bronchoscopy. Twenty three patients had bronchopulmonary Kaposi's sarcoma; occult alveolar haemorrhage was present in 16 of these (including two in whom no tracheobronchial lesions were evident at bronchoscopy, but in whom the diagnosis was confirmed at necropsy). Forty patients had other diagnoses including Pneumocystis carinii pneumonia and bacterial pneumonia; 18 had occult haemorrhage.

Occult alveolar haemorrhage seems to be a non-specific finding in HIV-1 positive men undergoing bronchoscopy.
\end{abstract}

Kaposi's sarcoma in human immunodeficiency virus (HIV) positive patients frequently affects the skin, lymph nodes, and gastrointestinal tract. Lesions may also occur in the lung ${ }^{12}$ and are a cause of pulmonary infiltrates, pleural effusions, and hilar and mediastinal lymphadenopathy. ${ }^{13}$ Bronchopulmonary Kaposi's sarcoma is often diagnosed at necropsy rather than by bronchial or transbronchial biopsy, ${ }^{1-4}$ and up to a third of cases are missed at bronchoscopy. ${ }^{14}$ Occult alveolar haemorrhage has frequently been described at necropsy and in open lung biopsy specimens from patients with bronchopulmonary Kaposi's sarcoma. ${ }^{45}$ The presence of haemosiderin laden macrophages in bronchoalveolar lavage fluid can be suggestive of occult alveolar haemorrhage caused by bronchopulmonary Kaposi's sarcoma. ${ }^{6}$ In this study we looked for evidence of occult alveolar haemorrhage in bronchoalveolar lavage fluid from HIV positive patients with bronchopulmonary Kaposi's sarcoma and with other respiratory diseases to evaluate whether this finding increases the yield of bronchoscopy and bronchoalveolar lavage for the diagnosis of bronchopulmonary Kaposi's sarcoma and discriminates between this and other diagnoses.

\section{Methods}

We studied 63 patients aged 27-60 (mean = 39) years who had presented with respiratory symptoms and abnormal chest radiographs; none had haemoptysis. All were male homosexuals and were known to be HIV-1 antibody positive or to have a diagnosis of AIDS at the time of investigation. In group 1 were 23 patients with bronchopulmonary Kaposi's sarcoma; all had cutaneous and palatal lesions of Kaposi's sarcoma. All underwent bronchoscopy to substantiate the diagnosis and to exclude infection. At bronchoscopy in 20 patients multiple, discrete, raised violaceous or red tracheobronchial lesions of Kaposi's sarcoma were seen; in the remaining three patients no lesions were seen. At necropsy (performed one to 26 (mean $=10.4$ ) weeks following bronchoscopy) in 14 patients, including the three without Kaposi's sarcoma seen at bronchoscopy, extensive violaceous tracheobronchial or parenchymal lesions of Kaposi's sarcoma, or both, were identified. In group 2 were 40 patients who had alternative diagnoses made by microbiological or cytological examination of bronchoalveolar lavage fluid or by subsequent percutaneous needle biopsy of the lung. None had cutaneous or palatal Kaposi's sarcoma and no bronchial lesions were seen at bronchoscopy. None developed cutaneous or palatal Kaposi's sarcoma over 12 weeks of follow up.

At bronchoscopy, following inspection of the bronchial tree, bronchoalveolar lavage was performed from the right lower or middle lobe in all patients using a previously described technique. ${ }^{7}$ Aliquots of lavage fluid were centrifuged for five minutes at $1500 \mathrm{rpm}$. Smears were made from the cell pellet and fixed in industrial methylated spirit. The smears were stained with Grocott's methenamine silver, Papanicolau, Gram and Ziehl-Neelsen stains to detect infections. Smears were also stained with Perls's iron stain, and one of us (GK) who did not know the clinical diagnosis, made a quantitative estimate of haemosiderin laden macrophages by counting the average number of haemosiderin laden macrophages in 10 high power fields ( $\times 400$ magnification).

The presence or absence of haemosiderin laden macrophages in bronchoalveolar lavage fluid from patients in the two groups was compared using $\chi^{2}$ analysis with Yates' correction for small numbers; a $p$ value of $<0.05$ was taken to be significant.

\section{Results}

The diagnoses in group 2 were Pneumocystis carinii pneumonia in 24 patients, bacterial pneumonia in 11 patients, Mycobacterium tuberculosis in two patients, disseminated Mycobacterium avium-intracellulare (in blood and bronchoalveolar lavage) in two patients and intrapulmonary lymphoma in one patient. The 
Haemosiderin laden macrophages per high power field in bronchoalveolar lavage fluid from patients with bronchopulmonary Kaposi's sarcoma and other diagnoses

\begin{tabular}{lllll}
\hline & \multicolumn{5}{c}{ Number of haemosiderin laden macrophages } \\
\cline { 2 - 5 } & Negative & $<10$ & $10-100$ & $>100$ \\
\hline Group 1 & & & & \\
Visible Kaposi's at bronchoscopy & 1 & 4 & 7 & 3 \\
No visible Kaposi's sarcoma & 7 & 4 & 1 & 1 \\
Total & & & & 4 \\
Group 2 & 13 & 6 & 2 & 3 \\
Pneumocystis carinii pneumonia & 8 & 1 & 2 & 0 \\
Bacterial pneumonia & 0 & 0 & 1 & 1 \\
Mycobacterium tuberculosis & 1 & 0 & 0 & $1^{\star}$ \\
Mycobacterium avium-intracellulare & 0 & 0 & 0 & 1 \\
Lymphoma & 22 & 7 & 5 & 6 \\
Total & & & & \\
\hline Pationt & &
\end{tabular}

$\star$ Patient was thrombocytopenic

haemosiderin laden macrophage scores for the patients in both groups are shown in the table.

Three patients with Kaposi's sarcoma and two patients with $P$ carinii pneumonia had platelet counts below $70 \times 10^{9} / 1$; all of these patients had negative haemosiderin scores. One of the patients with $M$ avium-intracellulare was also thrombocytopenic and had a haemosiderin laden macrophage score of more than 100 . In all patients the blood urea was less than $12 \mathrm{mmol} / \mathrm{l}$ and the International Normalised Ratio (INR) was less than 1.5. The presence of haemosiderin laden macrophages did not discriminate between those with bronchopulmonary Kaposi's sarcoma and other diagnoses: $\chi^{2}$ $=2 \cdot 67$; one degree of freedom; $p=$ NS.

\section{Discussion}

Occult alveolar haemorrhage was detected at open lung biopsy in all 12 patients with histologically confirmed bronchopulmonary Kaposi's sarcoma in one study, ${ }^{1}$ and in another evidence of occult alveolar haemorrhage was found at necropsy in patients with AIDS, bronchopulmonary Kaposi's sarcoma, and also bacterial pneumonia. ${ }^{5}$ Fouret et al found evidence of occult alveolar haemorrhage (by staining bronchoalveolar lavage fluid with Perls's stain to detect haemosiderin laden macrophages) in six of nine patients with bronchopulmonary Kaposi's sarcoma. This finding was thought to be highly specific for bronchopulmonary Kaposi's sarcoma as haemosiderin laden macrophages were not detected in bronchoalveolar lavage from 75 HIV-1 positive patients with other diagnoses including $M$ tuberculosis and cytomegalovirus, or negative investigations. ${ }^{6}$

We have shown that occult alveolar haemorrhage, detectable in bronchoalveolar lavage fluid, occurs in HIV positive patients with bronchopulmonary Kaposi's sarcoma and with other diseases. Of note were the three patients with bronchopulmonary Kaposi's sarcoma confirmed at necropsy, who had normal tracheobronchial anatomy at bronchoscopy. Two of these patients had haemosiderin laden macrophages in their lavage fluid, as did 18 of the patients with alternative diagnoses, including both patients with $M$ tuberculosis and the patient with lymphoma. However, only three of the 11 patients with bacterial pneumonia had evidence of occult alveolar haemorrhage.

It is concluded that the presence of haemosiderin laden macrophages in bronchoalveolar lavage fluid is a non-specific finding in HIV positive patients and does not discriminate between bronchopulmonary Kaposi's sarcoma and other diagnoses.

1 Ognibene FP, Steis RG, Macher AM, et al. Kaposi's sarcoma causing pulmonary infiltrates and respiratory failure in the acquired immunodeficiency syndrome. Ann Intern Med 1985;102:471-5.

2 Hanson PJV, Harcourt-Webster JN, Gazzard BG, Collins JV. Fibreoptic bronchoscopy in diagnosis of bronchopulmonary Kaposi's sarcoma. Thorax 1987;42:269-71.

3 Meduri GU, Stover DE, Lee M, Myskowski PL, Caravelli JF, Zaman MB. Pulmonary Kaposi's sarcoma in the acquired immunodeficiency syndrome. Am $f \mathrm{Med}$ acquired imm

4 Garay SM, Belenko M, Fazzini E, Schinella R. Pulmonary manifestations of Kaposi's sarcoma. Chest 1987;91: manife

5 Nash G, Fligiel S. Pathologic features of the lung in the acquired immunodeficiency syndrome (AIDS): an autopsy study of seventeen homosexual males. $A m \mathcal{F}$ Clin Pathol 1984;81:6-12.

6 Fouret PJ, Touboul JL, Mayaud CM, Akoun GM, Roland J. Pulmonary Kaposi's sarcoma in patients with acquired immunodeficiency syndrome: a clinicopathological study. Thorax 1987;42:262-8.

7 Griffiths MH, Kocjan G, Miller RF, Godfrey-Faussett P. Diagnosis of pulmonary disease in human immunodeficiency virus infection; role of transbronchial biopsy and bronchoalveolar lavage. Thorax 1989;44:554-8. 